\title{
STUDY ON EXTRACTING EDGE OF CROPLAND SCENERY
}

\author{
Mingxia Shen, Zhiye Yan, Changying Ji \\ Nanjing Agricultural University, Nanjing Agricultural University, 40\# Dianjiangtait Road, \\ Pukou Nanjiang, 210031, P. R. China, Tel: +86-025-58606585, Fax: +86-025-58606585
}

Abstract: Vision navigation and location is a main function in the vision system of intelligent agricultural mobile robot, and the edge feature of images is an important feature for vision navigation and location. According to the characteristic of cropland scenery, a compactly supported dyadic antisymmetric wavelet with respect to origin is brought forward to detect edges of cropland image. A set of filters were given that can be used to construct the edge detecting wavelet. The edge features are provided by determining the local maxim of wavelet coefficient at dyadic scale of the image. After the computer simulation was carried out on the cropland image, the continuous and smooth edge image can be got. The edges of cropland scenery are extracted accurately. The experiment result reveals that the method is efficient and practicable.

Keywords: Farm Crops, Marginal information, Wavelet transforms, Computer vision

\section{INTRODUCTION}

The detection of the edges in cropland scenery is the fundamental content of the wireless navigation system in mobile agricultural robot and it still remains an issue which has not been settled satisfactorily. In the common method of edge detection, one thing still stands in the way: the noises of the image are increased when the edges are maintained and enhanced, whereas

Shen, M., Yan, Z. and Ji, C., 2008, in IFIP International Federation for Information Processing, Volume 259; Computer and Computing Technologies in Agriculture, Vol. 2; Daoliang Li; (Boston: Springer), pp. 1411-1416. 
faintness was caused when the random noises are eliminated. We need to look for a kind of detection means which is insensitive to noise and can locate accurately, neither neglecting the real edges nor bringing about fake edges.

The edges points are usually located at where the grayness of the image mutates. Because wavelet transform is denoted by multi-resolution that can provide signals, it has good analytic characteristics of time-frequency localization and can meanwhile provide the time domain and frequency domain of the image signals. When detecting and locating the image edges, we can make use of the transmitting information between signals and resolution under every resolution, which features dual selectivity of space and frequency and can quantitatively describe the features of different structure boarders in the image. Therefore, this method can effectively detect and locate the edges of images and has been widely used in image processing and pattern recognition.

As far as the cropland scenery image is concerned, the boarders of some important structures in the image are usually some regular curves, which have strong internal geometrical characteristics. The grayness transformation in the curve direction is smooth while that in the vertical direction is singular. So the modular amplitude value, angle and position of the wavelet transformation in curve direction will not change notably. The random distributed boundary points in the image caused by noise will not bring about smooth singular curve; the maximal value point caused by noise is irregular at amplitude, angle and position. The various features of cropland scenery and noise provide the major reference for edge detection by using wavelet transform. Because the quality of wavelet edge detection is closely related with the features of wavelet function, this thesis brings forward a compactly supported dyadic antisymmetric wavelet with respect to origin to detect the edges in the image.

\section{MATIERIALS AND METHOD}

\subsection{The Principle of Wavelet Edge Detection}

Suppose $\theta(x, y)$ is an adequately smooth duality function, which satisfies the following conditions (Mallat S, 1992)

$$
\int_{-\infty}^{\infty} \int_{-\infty}^{\infty} \theta(x, y) \mathrm{d} x \mathrm{~d} y=1 \lim _{x^{2}+y^{2} \rightarrow \infty} \theta(x, y) \rightarrow 0
$$


Introduce signal $\theta_{s}(x, y)=\frac{1}{s^{2}} \theta\left(\frac{x}{s}, \frac{y}{s}\right)$

Define $\psi_{s}^{1}(x, y)=\frac{\partial \theta(x, y)}{\partial x} \psi_{s}^{2}(x, y)=\frac{\partial \theta(x, y)}{\partial y}$

Then function $\psi^{1}(x, y)$ and $\psi^{2}(x, y)$ are two-dimension wavelets.

Transforming the image by using dyadic wavelet and then detecting in the gradient direction the maximum point of the modular value and its corresponding revulsion point (singular point) of grayness in the image, we can get edge points, the amplitude of modular value and the strength of the edges. Thereafter, connecting the neighbor edge points that are of similar direction and modular value into one edge chain and eliminating the chain whose length is shorter than the threshold, we can get the edge of certain scale.

\subsection{The Structure of Compactly Supported Dyadic Antisymmetric Wavelet with Respect to Origin}

The dyadic expansion of $\hat{\psi}^{1}\left(\omega_{x}, \omega_{y}\right), \hat{\psi}^{2}\left(\omega_{x}, \omega_{y}\right)$ to cover the whole Fourier surface.

By further study we can know that in order to eliminate the influence of the edges and increase the operation speed, we can choose two wavelets (Yang Fusheng, 2000) $\psi^{1}(x, y), \psi^{2}(x, y)$, which can be expressed as the product of a univariate function with $x$ as the independent variable and that with $y$ as the independent variable, i.e., $\psi^{1}(x, y)=\psi(x) \xi(y), \quad \psi^{2}(x, y)=\xi(x) \psi(y), \quad$ in $\quad$ which $\quad \hat{\psi}(2 \omega)=G(\omega) \hat{\varphi}(\omega)$, $\hat{\xi}(2 \omega)=L(\omega) \hat{\varphi}(\omega), \psi(x)$ and $\xi(x)$ are the anti-symmetrical and symmetrical functions respectively at $x=0$. The symmetrical dyadic wavelet is the tool for detecting roof-style edges (Xu Peixia, 1996), whereas anti-symmetrical dyadic wavelet is that for step-lifting edges. By using both of them simultaneously, we can detect and classify all the edges in the cropland scenery. $\hat{\varphi}(\omega)$ is the Fourier transform of univariate scale function $\varphi(x)$, which can be expressed as $\hat{\varphi}(\omega)=\prod_{j=1}^{\infty} H\left(2^{-j} \omega\right), H(\omega) \in L^{2} \quad(-\Pi, \Pi),|H(\omega)| \leq 1$. $G(\omega), L(\omega)$ respectively refer to the Fourier transforms of digital filters $g(n), l(n)$. Then make $G(\omega), L(\omega)$ satisfy 


$$
\left\{\begin{array}{c}
G(\omega)=j \operatorname{sgn}(\omega)\left(1+\sum_{k=1}^{N} a_{k} \cos k \omega\right) \sqrt{1-|H(\omega)|^{2}} \in L^{2}(-\pi, \pi) \\
L(\omega)=\frac{1}{\sqrt{2}}\left(1+\sum_{k=1}^{T} b_{k} \cos k \omega\right) \sqrt{1+|H(\omega)|^{2}} \in L^{2}(-\pi, \pi)
\end{array}\right.
$$

in which $\left(a_{1}, a_{2} \ldots \ldots . a_{N}\right)$ is an optional $\mathrm{N}$-dimensional real-number group that satisfies $\sum_{k=1}^{N}\left|a_{k}\right|<1$, while $\left(b_{1}, b_{2} \ldots \ldots . b_{T}\right)$ is an optional P-dimensional realnumber group that satisfies $\sum_{k=1}^{T}\left|b_{k}\right|<1$. Then we have

$$
\sum_{j=\infty}^{\infty}\left[\left|\hat{\psi^{1}}\left(2^{j} \omega_{x}, 2^{j} \omega_{y}\right)\right|^{2}+\left|\hat{\psi^{2}}\left(2^{j} \omega_{x}, 2^{j} \omega_{y}\right)\right|^{2}\right]=1 .
$$

That means $\psi^{1}, \psi^{2}$ make up the basic dyadic wavelet group that detects the edges in the two-dimensional image of the cropland scenery. Set dual scale function $\varphi(x, y)=\varphi(x) \varphi(y)$, and we can get the wavelet transform of the image in the above dyadic wavelet group:

$$
\begin{aligned}
& S_{2}^{j} f(x, y)=\sum_{m \in Z} \sum_{n \in Z} h_{(m)} h_{(n)} S_{2}^{j-1} f\left(x-2^{j-1} n, y-2^{j-1} m\right) \\
& W_{2^{j}}^{j} f(x, y)=\sum_{m \in Z} \sum_{n \in Z} l_{(m)} g_{(n)} S_{2}^{j-1} f\left(x-2^{j-1} n, y-2^{j-1} m\right) \\
& W_{2^{j}}^{j} f(x, y)=\sum_{m \in Z} \sum_{n \in Z} g_{(m)} l_{(n)} S_{2}^{j-1} f\left(x-2^{j-1} n, y-2^{j-1} m\right)
\end{aligned}
$$

其中：

in which

$$
\begin{aligned}
& S_{2}^{j} f(x, y)=f(x, y) * \varphi_{2}^{j}(x, y), \quad W_{2^{j}}^{1} f(x, y)=f(x, y) * \psi_{2^{j}}^{1}(x, y) \\
& W_{2^{j}}^{2} f(x, y)=f(x, y) * \psi_{2^{j}}^{2}(x, y) .
\end{aligned}
$$

\subsection{The Choosing of Compactly Supported Dyadic Antisymmetric Wavelet with Respect to Origin and the Analysis of the Characteristics of the Filter}

From the structure of the above edge detection wavelet, we can see that choosing the edge detection wavelet equals to choosing digital filters $g(n)$ 
and $l(n)$. Because the edge detection of the cropland scenery demands that the detected edges have good locality (i.e., correct edge location) and no position excursion, digital filter group must have linear phase and should have symmetry about zero point (Zhang Guobao, 1998).

In order to detect the local maximum modular value of the image and evaluate the gradient value of the grayness variation, we choose high pass filter $g(n)$ as odd symmetry, i.e., $g_{(n)}=-g_{(-n)}$ and $g_{0}=0$, then the Fourier transform is $G(\omega)=\sum_{n \in Z} g_{(n)} e^{-j n \omega}$, in which $G(\omega)$ is real function and $G(-\omega)=-G(\omega)$.

Because low-pass filter $l(n)$ has smoothening function, it can't have odd symmetry and can only have even symmetry, i.e., $l_{(n)}=l_{(-n)}$, and its Fourier transform is $L(\omega)=\sum_{n \in z} l_{(n)} e^{-j n \omega}$, in which $L(\omega)$ is real function and $L(\omega)=L(-\omega)$.

Calculate the filtering coefficient $g_{n}, l_{n}$ and we can get the following table:

\section{THE RESULT AND ANALYSIS OF THE EXPERIMENT}

Adopt the filter group proposed in this thesis, wavelet transform the tested image by using the filtering coefficients listed in Table 1; adopt the method put forward in this thesis to detect the local maximum modular value;

Table 1. Dyadic wavelet transform filter coefficient

\begin{tabular}{cccccc}
\hline & 0 & 1 & 2 & 3 & 4 \\
\hline $\mathrm{g}_{\mathrm{n}}$ & 0 & 0.0506770 & 0.180425 & 0.025216 & -0.003602 \\
$\mathrm{l}_{\mathrm{n}}$ & 0.190682 & 0.066190 & 0.031165 & 0.007269 & 0.000035 \\
& 0 & -1 & -2 & -3 & -4 \\
$\mathrm{~g}_{\mathrm{n}}$ & & -0.506770 & -0.180425 & -0.025216 & 0.003602 \\
$\mathrm{l}_{\mathrm{n}}$ & & 0.066190 & 0.031165 & 0.007269 & 0.000035 \\
\hline
\end{tabular}


assume the points of the local maximum modular values that are greater than the wavelet transform modular value mean as the edge points; repeat the above operations on the given images, and connect the edge points in the 8neighborhood according to the 8 connection rule; assume the threshold value and eliminate the chains who are less than the threshold value, because they might be caused by noises, and then we obtain the edge image.

The thesis is based on the compactly supported dyadic antisymmetric wavelet with respect to origin to detect the edges in the cropland images. We can see that the detected edge in the image is of fine locality and is abundant in details-every edge has been extracted. This shows that the wavelet put forward in the thesis is of the same quality in each direction and all the edges are continuous and of mono picture elements. Because the selected high-pass filter is symmetric about the zero point, the detected edges in the image have no translation of picture elements and are of accurate localization.

The experiment proves: if we use a wavelet transform of a certain scale to detect the fine edges in the cropland image, we should choose small neighborhood; if to detect the main edges, we should choose appropriate neighborhood. The experiment has also provided a thought for detecting various edges caused by different revulsion points, such as shadows, oscillations, peaks and grains.

\section{REFERENCES}

Mallat S, Whwang W. Singularity Detection and Processing with Wavelets, IEEE Trans. Information Theory, 1992, 38(20):617-643

Xu Peixia, Sun Gongxian, Wavelet analysis and applicable examples, China Science and Technology University Press, 1996, 21-38

Yang Fusheng. The engineering analysis and application of wavelet transform. Science Press, 2000, 112-176

Zhang Guobao, Chen Weinan. Multi-scale edge extraction based on orthogonal wavelet transform, China Image and Graph Learned Journal, 1998, (8):651-654, 38(20):617-643 